\title{
THE TREATMENT OF TICS IN CHILDHOOD
}

\author{
A REVIEW AND A FOLLOW-UP STUDY \\ BY \\ DAVID M. ZAUSMER \\ From the Department of Studies in Psychological Medicine, University of Liverpool
}

(RECEIVED FOR PUBLICATION JULY 8, 1954)

Tics are involuntary movements of the skeletal musculature involving functionally related muscle groups. They are sudden, unexpected, brief contractions, of variable intensity, repeated at irregular intervals, and occur in descending order of frequency from the cephalic to the caudal extremity. They increase in severity during emotional excitement, decrease as a result of distraction or concentration, and disappear during sleep. Tics can be interpreted as biologically purposeful (protective, defensive, or offensive) but serve no apparently useful purpose since their objective is non-existent. In adults they are single and localized, whereas in children they are multiple and migratory. Tics are seen more commonly in boys than in girls and in children than in adults.

In childhood they are found in association with a variety of psychiatric and organic disorders and may occur as a sequel to chorea (Creak and Guttman, 1935) and epidemic encephalitis (Wilson, 1927); in rare cases they are accompanied by echophenomena and coprolalia (Gilles de la Tourette, 1885).

I have not used 'habit spasm', the popular alternative term for tic, because, strictly speaking, a tic is neither a habit (Garrod, Batten and Thursfield, 1934) nor a spasm (Wilson, 1940).

The first systematic treatise on tics was written by Meige and Feindel (1902) and included a comprehensive account of the many methods employed at the turn of the century. A large bibliography has accumulated since then and a very great variety of treatments has been recommended. Sedatives, particularly bromides, as well as 'tonics' have both constantly been advised, though Hobhouse (1939) is one of the few who state categorically that drugs have no place in the treatment of tics; improved hygiene by such measures as fresh air, wholesome diet and change of environment; rest, ranging from bed rest to convalescence; eradication of sources of local irritation or local sepsis, including removal of tonsils and adenoids; various forms of physiotherapy at one time popular and now almost obsolete; advice about school attendance has been contradictory.

The use of suggestion and hypnosis has had few supporters apart from Russell (1910), Fleming (1912), Hassin (1925), Wilson (1927), Brain (1928) and Lust (1930).

Most writers have agreed that some form of psychological guidance is indicated and the procedures recommended have varied from simple advice to prolonged psycho-analysis. The introduction of the psycho-analytical methods of treatment into child psychiatry has influenced both the understanding and the treatment of the tiqueur (Sadger, 1914; Ferenczi, 1926; Deutsch, 1925; Wilder and Silbermann, 1927; Menaker, 1945; Klein, 1948; Mahler, 1949).

Very recent methods such as brief psychotherapy, narco-analysis and group psychotherapy have been employed in selected cases (Lebovici, 1952).

\section{Present Study}

This paper forms part of an investigation into the nature, treatment and prevention of tics in childhood which began in May, 1952. It is an attempt to appraise the results of treatment of 96 consecutive tiqueurs referred to the Psychiatric Department of the Royal Liverpool Children's Hospital since May, 1947. These comprise 53 cases investigated in the department before May, 1952, called Group I, and 43 cases investigated by me after that date, called Group II.

The proportion of new cases seen each year up to May, 1953, is indicated in Table 1. The small number of tiqueurs treated in 1947 cannot be accounted for; the large number referred after May, 1952, is directly attributable to the interest shown in this research project. The mean annual referral rate before that date was $5 \%$ of the total number of cases, which included every variety of psychiatric disorder in childhood. 
It will be appreciated that the treatment of tics in childhood is not solely a psychiatric problem, since all of these patients were sent to hospital by general practitioners, and $62 \%$ were referred to the Psychiatric Department by paediatricians.

TABLE 1

\section{ANNUAL REFERRAL RATE OF NEW TIC CASES}

\begin{tabular}{ccc}
\hline Year of Referral & Total Number of Cases & Number of Tic Cases \\
\cline { 2 - 3 } \cline { 3 - 3 } 1947 & 178 & $31 \%(1 \%)$ \\
1948 & 156 & $11(7 \%)$ \\
1949 & 195 & $16(7 \%)$ \\
1959 & 233 & $11(6 \%)$ \\
1951 & 192 & $29(17 \%)$ \\
$1952-53$ & 173 & \\
\hline
\end{tabular}

\section{Description of the Sample}

The terminology of Barton Hall (1947) has been used throughout this investigation in order to maintain continuity in the recording and assessment of the clinical data.

The following features of the sample studied are considered to be representative of tiqueurs in general, by comparison with cases made available from other sources.

Sex Incidence. There were 63 boys and 33 girls, that is, a ratio of approximately $2: 1$.

Intelligence Rating. The intelligence quotients (I.Q.s) of 89 patients tested by the same psychologist on the Revised Stanford-Binet Scale ranged from 58 to 154 (mean $=106 \cdot 1$, S.D. $\pm 21 \cdot 2$ ) with an approximately normal distribution.

Age at Onset. This was difficult to ascertain accurately in some cases and was therefore estimated to the nearest half-year. The ages ranged from
$3 \frac{1}{2}$ to $12 \frac{1}{2}$ years with a well-defined peak at 7 years; five tiqueurs were under 5 years at the onset.

Duration of Symptoms. Of the patients $34.4 \%$ were examined within six months and $56.3 \%$ within a year of onset. The longest interval that elapsed between the onset and referral to a psychiatrist was eight years.

Personality and Body-build. These tiqueurs had many personality traits in common; in descending order of frequency they were restless, sensitive irritable, stubborn, excitable, phobic, apprehensive and quick-tempered. With few exceptions they were asthenic, wiry children; in no case was the pseudo-Fröhlich habitus, as described by Mahler, Luke and Daltroff (1945), seen.

Sibling Status. The number of siblings per family ranged from one to eight with an average of three. Forty tiqueurs $(42 \%)$ were eldest children, $22(23 \%)$ were youngest, $11(11 \%)$ were only children, and $23(24 \%)$ were middle children. The equivalent figures of Mahler's series of 33 cases show little correspondence (eldest $30 \%$, youngest $24 \%$, only child $36 \%$, and middle child $9 \%$ ).

Parents. The majority of parents were anxious, restrictive and rigid; this was not attributable to their advanced age, as suggested by Latimer (1945). The conflicting personalities of parents and tiqueur, and in particular the disturbed mother-child relationship, was found to be a major source of anxiety in the patient.

Family History. The importance of a psychopathic heredity in the aetiology of tics has been stressed repeatedly in the literature, but few and inadequate statistics have been published. Table 2

TABLE 2

FAMILIAL INCIDENCE OF PSYCHOPATHY AND OTHER DISORDERS*

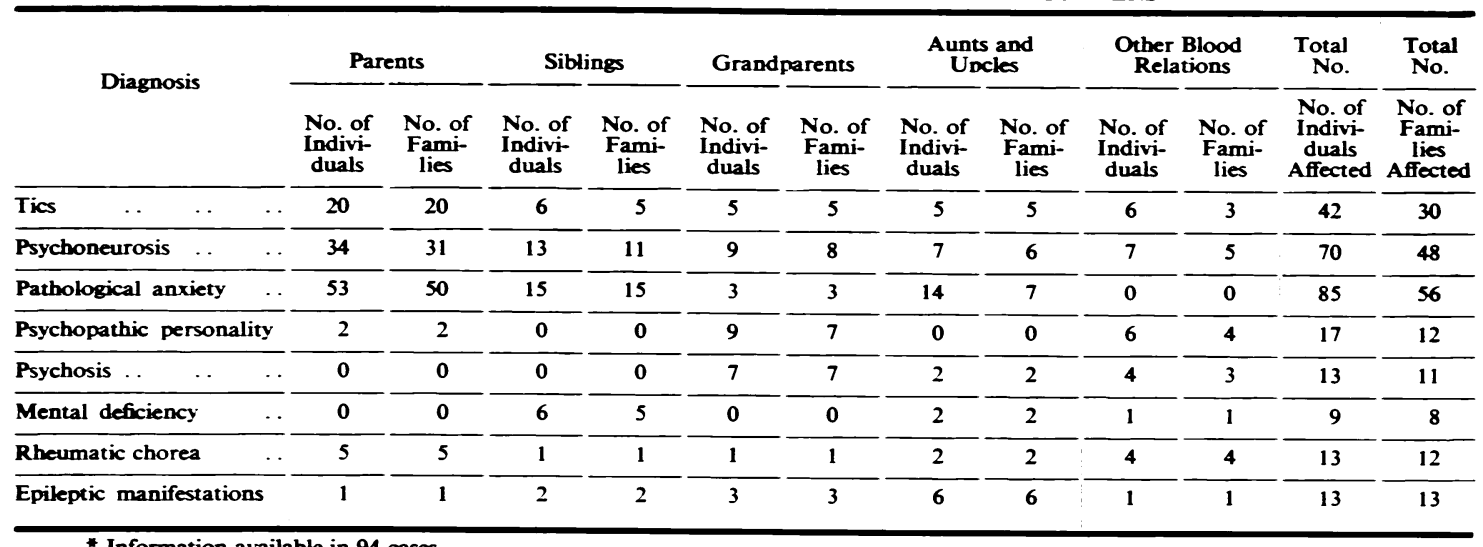

* Information available in 94 cases. 
summarizes the familial incidence of psychopathy and neuropathy in this sample and indicates the relatively high proportion of parents with pathological anxiety, psychoneurosis and tics. The adverse influence of such parents on the susceptible child tiqueur will be readily appreciated.

\section{Treatment and Results in Group I}

Of the 53 patients investigated before May, 1952 , 42 were seen two or three times for clinical investigation, mental testing and parental guidance, and 11 attended the Liverpool Psychiatric Clinic over a period of five to 33 weekly sessions for more prolonged psychotherapy.

In 17 cases the parents were reassured about the child's physical and mental health. A simple explanation of the cause of the tics was given in 35 cases. The majority of parents were instructed to ignore the tics, and 37 were offered advice depending on the nature of the individual problems. Seven children presented school problems, and convalescence was arranged in two cases.

Four patients were referred to the ophthalmologist, three with errors of refraction and one with strabismus. Two cases with nasal symptoms and one with intermittent deafness received appropriate treatment. Two patients were referred to the paediatricians for investigation of abdominal pain and treatment of migraine-ketosis attacks. In three cases the general practitioners were requested to observe the child on account of a rheumatic diathesis. Only one child in the whole series was treated with phenobarbitone.

Results. The results of treatment in this first group were evaluated by means of a follow-up inquiry. Forty-nine of the 53 patients were traced, and of these I re-examined 39, eight were interviewed by a psychiatric social worker, and information about the remaining two was obtained by correspondence.

The information was sufficiently accurate for statistical analysis in only 41 cases, but since they appeared to be fairly representative of the total sample, it is doubtful whether the remaining cases would have influenced the findings to any appreciable degree.

The severity of the tics throughout the investigation was rated on a six-point scale according to a clinical estimate of the frequency, irrespective of the number of concurrent tics:

$(5+)$ Tics repeated continuously every day for a few weeks.

(4+) Tics repeated intermittently every day for a few weeks.
(3+) Tics recurring spasmodically, at intervals of a few days, for a few weeks.

$(2+)$ Tics recurring for a few days at intervals of a few weeks.

$(1+)$ Tics recurring for a few days two or three times a year during periods of marked stress or excitement.

(0) Complete absence of tics for one year.

Progress of Tics. The severity of the tics at follow-up is shown in Table 3 . There was a marked improvement as a whole, although only 10 cases $(24 \%)$ had been completely free of tics for at least a year.

The duration of the tics in the 10 recovered cases ranged from three to eight and a half years (mean = four and a half years); the duration in the other 31 cases followed up ranged from one and a half to eleven years (mean $=$ four and a half years). It is apparent that severe tics persist for several years, contrary to the opinions expressed in many textbooks as to their short duration.

Taking as the criterion of improvement a change of 2 - or more, 31 cases $(76 \%)$ were improved at followup and $10(24 \%)$ unimproved. Of the 34 patients who received brief treatment, 24 were improved, and of the seven patients who received more prolonged psychotherapy, six were improved. Thirteen patients were considered to require further treatment when re-examined; of these only one had had intensive psychotherapy. As a result of the additional psychotherapy, nine patients improved, one was unimproved, and three failed to complete the treatment.

The relationship between progress at follow-up, on the one hand, and sex, intelligence, age at onset, duration before treatment, length of follow-up, age at follow-up, and severity of anxiety, on the other, were considered worthy of further investigation (see Table 3).

SEX. Of the 31 tiqueurs who were improved, there were 20 boys and 11 girls; and of the unimproved there were 10 boys and no girls. The sex difference is significant at the $5 \%$ level $(p=0.027)$; that is, the prognosis appears to be better in girls than in boys.

There was no statistically significant relationship between improvement and intelligence rating, age of onset or duration before treatment.

LENGTH of Follow-UP. The average follow-up interval of the improved group was 34.6 months, and that of the unimproved group, 26.7 months. The difference is almost significant at the $5 \%$ level $(\mathrm{t}=1 \cdot 743$, D.F. $=39,0 \cdot 1>\mathrm{p}>0.05)$. This suggests that the longer the interval after treatment, the more improvement can be expected. 
TABLE 3

RESULTS OF FOLLOW-UP OF CASES IN GROUP I

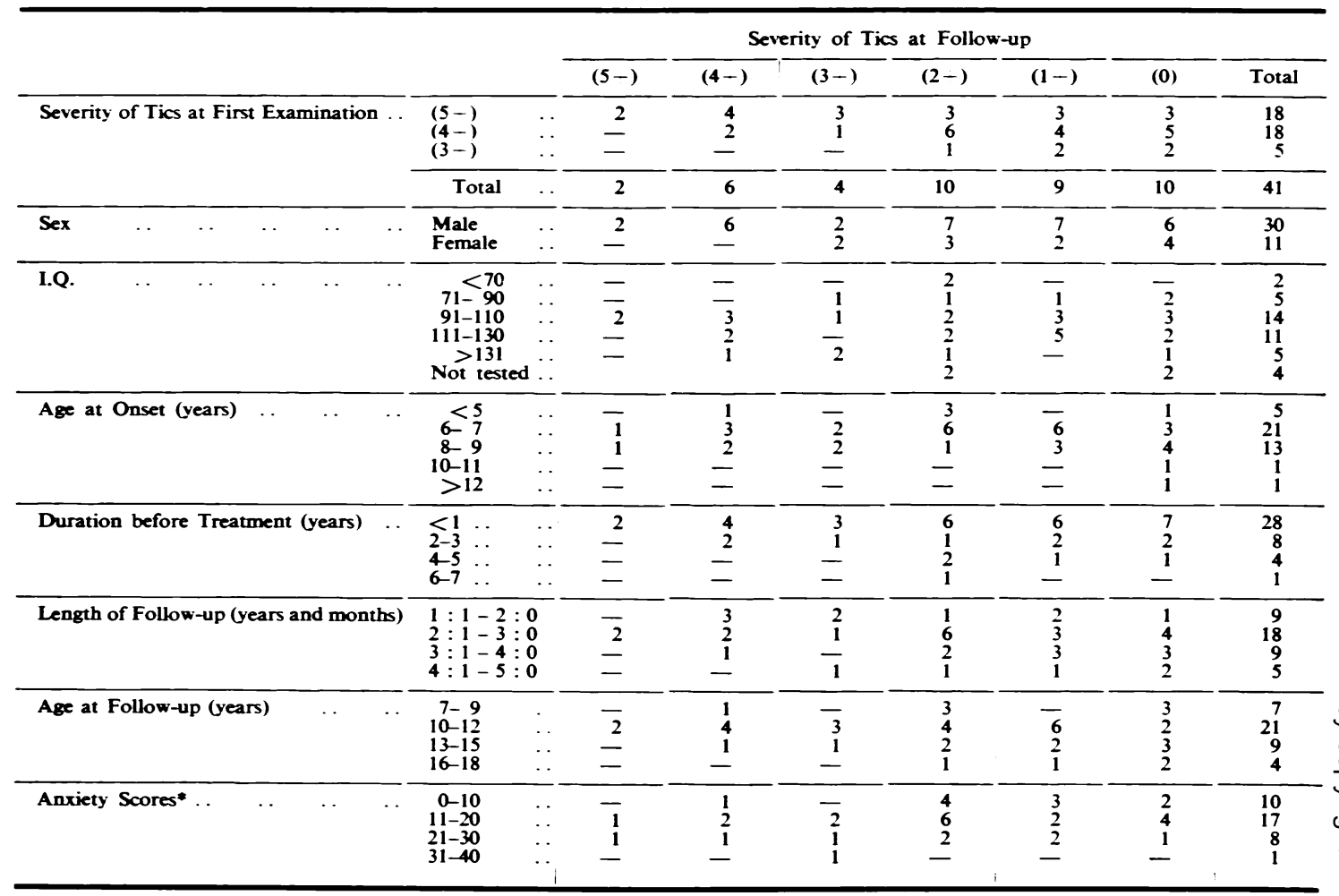

- In five cases data could not be scored accurately.

Age At Follow-ur. The difference between the average ages of the improved and unimproved groups (12:2 and 11:0 years, respectively) was not statistically significant $(t=1 \cdot 261$, D.F. $=39$, $0 \cdot 3>p>0 \cdot 2$ ). However, all six tiqueurs aged over 15 were improved, as compared with 25 improved of the remaining 35 . The difference in proportions improved, although not significant at the $5 \%$ level $(p=0 \cdot 164)$, is such as to suggest that tics tend to clear up by puberty. A more prolonged and extensive study is required to verify this conclusion.

INCIDENCE of ANXIETY. A frequent association between tics and symptoms of anxiety and tension was observed clinically and was confirmed by calculating the frequencies of 17 symptoms included under the following headings: (1) sleep disturbances, (2) tension habits, (3) motor unrest, (4) phobias, worry and poor concentration. The proportion of patients exhibiting the four groups of symptoms was $81,92,86$ and $83 \%$ respectively.

A disconcerting observation at follow-up was that the anxiety symptoms tended to be unaffected by treatment. In order to confirm this, each symptom was graded as to severity on a three-point scale, as described by Miles, Barrabee and Finesinger (1951). The arithmetical sum of the ratings for each patient was termed the 'anxiety score'. Expressing this score as a percentage of the possible total it was found that the severity of the four groups of symptoms was almost identical in the two corresponding samples of treated former and untreated current cases (viz. 22, 27, 41, 35\%, and 23, 22, 43, 30\% respectively).

It was further shown that improvement in the tics was unrelated to the severity of the anxiety by comparing the anxiety scores of the improved and unimproved groups. The difference between the means (13.4 and 17.3) was not statistically significant $(t=1 \cdot 250$, D.F. $=34,0 \cdot 3>p>0 \cdot 2)$.

\section{Treatment and Results in Group II}

Of the 43 patients examined after May, 1952, 19 were seen less than four times for investigation, mental testing and brief psychotherapy, and 24 
received more prolonged treatment over five to 29 sessions. The number of interviews was roughly proportional to the severity of the case.

Brief Psychotherapy. A minimum of two interviews was devoted to taking a history, physical and mental examination of the patient, and parental guidance. The informant (usually the mother) was encouraged to ventilate her anxieties, and the confidence of the patient was gained by showing an interest in both his health and in his worries, ambitions, and problems of home and school adjustment.

In every case the parent was reassured concerning the physical and mental state of the child. The tic was interpreted as a safety-valve for tension resulting most commonly from over-correction and restriction. The parent was advised against drawing the patient's attention to the movement and of the futility of punishment. Encouragement of normal activities and interests was advocated. Convalescence was recommended in two cases only in order to remove the patient from a disturbed home, and continued attendance at school was advised except when there was associated physical ill-health.

Physical treatment was required in a small number of cases for threadworms, errors of refraction, dental caries, sinusitis, septic tonsils, enlarged adenoids and otitis media. It was my impression that these sources of irritation aggravated the tics, possibly by lowering the threshold of neuromuscular excitability, and that physical treatment resulted in some degree of improvement. Drugs were not prescribed unless indicated for a complicating physical ailment.

Prolonged Psychotherapy. In the most severe cases treatment was continued at the Liverpool Psychiatric Clinic at weekly intervals. The relationship with the therapist enabled the patients to work through their emotional disturbances by means of drawing, painting, modelling and play techniques, which were selected according to the age, intelligence and symptomatology of the child.

The parents were interviewed at each visit to discuss the patient's progress and management. Emphasis was placed on the harmonious interaction of mother and child as the primary object of treatment. When this was achieved there was invariably an improvement in the patient's clinical condition, and it illustrated convincingly to the mother that the child's recovery depended to a large extent upon her own ability to maintain a happy relationship. It was noteworthy that the patient's progress fluctuated according to the mother's state of health, and in a few cases the mothers were advised to seek treatment for their own emotional disturbances.

Parental Group Psychotherapy. The improvement resulting from correction of parental mishandling was brought out by the following experiment.

Six mothers were invited to form a discussion group and met for one hour each week over a period of 14 weeks during which time the patients received no treatment. Initially common problems were presented for discussion and later individual difficulties concerning the handling of their children were raised and debated. I played an increasingly passive role at these meetings, allowing the parents to advise and criticize each other and intervening only in order to control the discussion. The subsequent modification of parental attitude was acknowledged by every member of the group, and the improvement in the tics was sufficiently pronounced in every case to exclude the possibility of spontaneous remission.

Results. Insufficient time has elapsed to evaluate the final results of treatment but information is available as to the progress of 34 of the 43 current cases observed over a period of one to 20 months. The remaining nine patients comprised the least severe cases in the series and had been given a good prognosis. Of the 34 cases, 28 showed improvement in the severity of the tics. Ten had been treated briefly and of these eight were improved; of the 24 who had received more prolonged treatment, 20 were improved.

Of the 34 patients followed up, the anxiety manifestations were improved in 29 cases. Nine of the 10 patients who had been treated briefly were improved, and of the 24 who had been treated over a longer period, 20 were improved.

\section{Discussion}

The apparent confusion in the treatment of tics over the past half-century is the result of the changing concepts of aetiology and of the different aims of therapy. The aetiology remains obscure, but, as a tentative hypothesis, it is suggested that tics in childhood are motor manifestations of psychological and possibly physiological, tension occurring in certain constitutionally predisposed individuals. The underlying source of the tension is attributed to the accompanying psychiatric and organic disorders, and it follows that treatment should deal primarily with the major disorder and only secondarily with the resulting symptoms.

This research has shown that the role of the mother is of paramount importance in the psychogenesis and 
treatment of tics. A study of the emotional development of the tiqueur has revealed an over-dependence on the mother throughout the prepubertal period. Maternal anxiety is readily conveyed to the nervous child and disharmony in the mother-child relationship has been identified frequently as a source of emotional conflict in the patient. In the majority of cases modification of parental attitude, increased maternal insight, and attention to the mother's health have brought about improvement in the tics and anxiety symptoms.

It is of interest that a comparison of the outcome of treatment of the former and current cases, that is, two equivalent samples treated along similar lines by different therapists, showed a similar rate of improvement in the frequencies of tics irrespective of the length of treatment. Although the findings are not strictly comparable owing to the large differences between the follow-up periods, they indicate that prolonged psychotherapy is not essential for the successful symptomatic treatment of tics.

It is of some practical importance that the assessments of severity and improvement by the parents were far less stringent than those described here. It was frequently noted that tic-ratings below $(4-)$ tended to be ignored or regarded as unimportant, a change of 1 - was accepted as a marked improvement, and of $2+$ as a complete recovery.

An analysis of the symptoms presented by these cases has revealed that tics are correlated most highly with manifestations of anxiety and tension. The tic-frequencies are apparently unrelated to the severity of anxiety symptoms and the latter tend to persist longer than the tics. It has also been found that mild anxiety symptoms improve with brief psychotherapy but severe and multiple anxiety symptoms respond only to prolonged psychotherapy. It is concluded therefore that psychotherapy is the primary treatment of tics in childhood and that the duration should be determined by the severity of the associated anxiety manifestations.

\section{Summary}

The literature on the treatment of tics in childhood over the past $\mathbf{5 0}$ years is reviewed.

An evaluation of the treatment of a consecutive series of 96 hospital cases by brief and prolonged psychotherapy is described. Fifty-three former patients were followed up after an interval of one to five years; the remainder were investigated and treated by the writer. The results indicate that each form of treatment has been equally effective in reducing the frequency of the tics.

A frequent association between tics and symptoms of anxiety and tension has been observed. Mild anxiety manifestations have improved with brief psychotherapy but severe emotional disturbances have required prolonged psychotherapy.

The significance of the mother-child relationship in the aetiology and treatment of tics is stressed.

I wish to express my gratitude to Dr. S. Barton Hall for his guidance and constant encouragement in this research, and to Dr. M. Barton Hall for her considerable help and advice in the preparation of this paper. My thanks are due to Mrs. O. L. Keidan for her assistance with the follow-up investigation, to Miss L. P. Gilpin for the mental testing, and to Mr. R. L. Plackett for guidance with the statistics.

\section{ReFERENCES}

Brain, W. R. (1928). Lancet, 1, 1295

Creak, M. and Guttmann, E. (1935). J. ment. Sci.. 81. 834.

Deutsch, H. (1925). Int. Z. Psychoanal., 11, 325.

Ferenczi, S. (1926). Further Contributions to the Theory and Technique of Psychoanalysis, p. 142. London.

Fleming, R. A. (1912). A Short Practice of Medicine. 2nd ed., pp. 775-7. London.

Garrod, A. E., Batten, F. E. and Thursfield, H. (1934). Diseases of Children, 3rd ed., pp. 690-3. Ed. Thursfield. H. and Paterson, D. Lildren, 3ro

Hall, M. B. (1947). Psychiatric Examination of the School Child, pp. 183-5. London.

Hassin, G. B. (1925). In Abt's Pediatrics, vol. 7. pp. 288-95.

Hobhouse, N. (1939). In A Survey of Child Psychiatry, pp. 235-45. Ed. Gordon, R. G. London.

Klein, M. (1948). Contributions to Psycho-analysis, 1921-1945, pp. 117-39. London.

Latimer, R. H. (1945). Nerv. Child., 4, 353.

Lebovici, S. (1952). Les Tics chez L Enfant. Paris.

Lust, F. (1930). The Treatment of Children's Diseases, trans. of 6th Germ. ed. by Levinsohn, S. A.. p. 247. Philadelphia.

Mahler, M. S. (1949). Psychoanal. Study Child., 3-4. 279.

, Luke. J. A. and Daltroff, W. (1945). Amer. J. Orthopsychiat., 15, 631 .

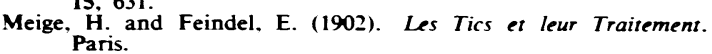

Menaker, E. (1945). Nerv. Child., 4. 335

Miles, H. H. W., Barrabee, E. L. and Finesinger, J. E. (1951). Psychosom. Med., 13, 83

Russell, J. S. R. (1910). In Allbutt and Rolleston's 4 System of Medicine, vol. 8, 2nd ed., pp. 614-38. London.

Sadger, J. (1914). Int. Z. Psychoanal., 2, 354.

Tourette, G. de la (1885). Arch. Neurol., Paris, 9. 19.

Wilder, J. and Silbermann, J. (1927). Beiträge zum Ticproblem. Berlin.

Wilson, S. A. Kinnier (1927). J. Neurol. Psychopath. 8. 93.

(1940). Neurology, vol. 2. pp. 1629-37. London. 